\title{
CYP3A4 mutation causes vitamin D-dependent rickets type 3
}

\author{
Jeffrey D. Roizen, ${ }^{1}$ Dong Li, ${ }^{2}$ Lauren O'Lear, ${ }^{1}$ Muhammad K. Javaid, ${ }^{3}$ Nicholas J. Shaw, ${ }^{4}$ Peter R. Ebeling, ${ }^{5}$ Hanh H. Nguyen, ${ }^{5}$ \\ Christine P. Rodda, ${ }^{6}$ Kenneth E. Thummel, ${ }^{7}$ Tom D. Thacher, ${ }^{8}$ Hakon Hakonarson, ${ }^{2}$ and Michael A. Levine ${ }^{1}$ \\ 'Division of Endocrinology and Diabetes and 'Center for Applied Cenomics, The Children's Hospital of Philadelphia (CHOP), University of Pennsylvania Perelman School of Medicine, Philadelphia, \\ Pennsylvania, USA. ${ }^{3}$ Nuffield Department of Orthopaedics, Rheumatology and Musculoskeletal Sciences, University of Oxford, Oxford, United Kingdom. ${ }^{4}$ Department of Endocrinology and Diabetes, \\ Birmingham Children's Hospital and Institute of Metabolism and Systems Research, University of Birmingham, Birmingham, United Kingdom. ${ }^{5}$ Department of Medicine, School of Clinical Sciences, \\ Monash University, Clayton, Victoria, Australia. ${ }^{6}$ Australian Institute for Musculoskeletal Science, Sunshine Hospital, and Department of Paediatrics, University of Melbourne,Parkville, Victoria, Australia. \\ 'Department of Pharmaceutics, University of Washington, Seattle, Washington, USA. ${ }^{8}$ Department of Family Medicine, Mayo Clinic, Rochester, Minnesota, USA.
}

\begin{abstract}
Genetic forms of vitamin D-dependent rickets (VDDRs) are due to mutations impairing activation of vitamin D or decreasing vitamin $\mathrm{D}$ receptor responsiveness. Here we describe two unrelated patients with early-onset rickets, reduced serum levels of the vitamin $D$ metabolites 25-hydroxyvitamin $D$ and 1,25-dihydroxyvitamin $D$, and deficient responsiveness to parent and activated forms of vitamin D. Neither patient had a mutation in any genes known to cause VDDR; however, using whole exome sequencing analysis, we identified a recurrent de novo missense mutation, c.902T>C (p.I301T), in CYP3A4 in both subjects that alters the conformation of substrate recognition site 4 (SRS-4). In vitro, the mutant CYP3A4 oxidized 1,25-dihydroxyvitamin D with 10-fold greater activity than WT CYP3A4 and 2-fold greater activity than CYP24A1, the principal inactivator of vitamin D metabolites. As CYP3A4 mutations have not previously been linked to rickets, these findings provide insight into vitamin $\mathrm{D}$ metabolism and demonstrate that accelerated inactivation of vitamin $\mathrm{D}$ metabolites represents a mechanism for vitamin $\mathrm{D}$ deficiency.
\end{abstract}

\section{Introduction}

Vitamin D deficiency rickets is a childhood disorder associated with impaired growth and skeletal mineralization. In most cases, vitamin D deficiency is due to inadequate exposure to sunlight and/or insufficient dietary vitamin D (1). Vitamin D is biologically inactive and undergoes 2 enzymatic conversions to become fully active 1,25-dihydroxyvitamin $\mathrm{D}$. The existence of rickets in areas with abundant sunlight has led to the identification of genetic forms of vitamin D-dependent rickets (VDDRs). Type 1 VDDRs are caused by defects in vitamin D activation, owing to mutations in the genes encoding either the renal 1- $\alpha$ hydroxylase (CYP27B1: VDDR-1A) (2) or the hepatic 25-hydroxylase (CYP2R1: VDDR-1B) $(3,4)$. Type 2 VDDRs are caused by defects in vitamin $\mathrm{D}$ receptor signaling due to mutations in the gene encoding the vitamin $\mathrm{D}$ receptor (VDR: VDDR-2A); or heterogeneous nuclear ribonucleoprotein C (HNRNPC: VDDR-2B) (5, 6), a VDR coactivator. Vitamin D homeostasis is also regulated by renal 24-hydroxylase (CYP24A1), an enzyme responsible for 24-oxidation of 1,25-dihydroxyvitamin $\mathrm{D}_{3}$ to inactive calcitroic acid (7) and degradation of 25 -hydroxyvitamin $\mathrm{D}_{3}$ to inactive 24,25-dihydroxyvitamin $\mathrm{D}_{3}$ (8). Here we describe a third kind of VDDR (VDDR-3), in which a gain-of-function mutation in

Authorship note: JDR and DL are co-senior authors.

Conflict of interest: The authors have declared that no conflict of interest exists.

Submitted: November 14, 2017; Accepted: February 13, 2018.

Reference information: J Clin Invest. 2018;128(5):1913-1918.

https://doi.org/10.1172/JCI98680.
CYP3A4, which encodes a P450 enzyme that metabolizes many xenobiotics and drugs, leads to vitamin D deficiency through accelerated vitamin D metabolite inactivation.

\section{Results and Discussion}

Clinical and biochemical studies. We studied 2 unrelated females from non-consanguineous families. Both subjects were born fullterm with normal birth weight and length. Proband 1.1 presented to medical attention prior to age 2 years with a history of bowed legs and unsteady gait noted at 20 months. Her pediatrician diagnosed rickets on the basis of bilateral genu varum, poor growth, and reduced serum calcium and phosphorus with elevated serum alkaline phosphatase and parathyroid hormone (Table 1). She was of European ancestry and lived in a sunny climate in Australia. At age 2.5 years she was referred for endocrine evaluation, at which time her height was $81.3 \mathrm{~cm}$ (6th percentile) and weight was 11.5 $\mathrm{kg}$ (35th percentile). Proband 2.1 was of Middle Eastern descent and did not walk until 4.5 years of age. She was treated for rickets in Jordan, Spain, and the Netherlands prior to evaluation in the United Kingdom at age 16 years, when she had a height of $154.3 \mathrm{~cm}$ (9th percentile). Wrist radiographs in both subjects showed features consistent with active rickets (Figure 1A). Both subjects had detectable serum vitamin $D_{3}$ but low serum levels of 25-hydroxyvitamin D and 1,25-dihydroxyvitamin D (Table 1), which increased only after administration of very large doses of vitamin $\mathrm{D}$ or calcitriol and declined rapidly thereafter. After oral administration of $50,000 \mathrm{IU}$ of vitamin $\mathrm{D}_{3}$, proband 1.1 showed a normal increase in serum cholecalciferol, indicating normal absorption of vita- 


\section{Table 1. Biochemical analyses}

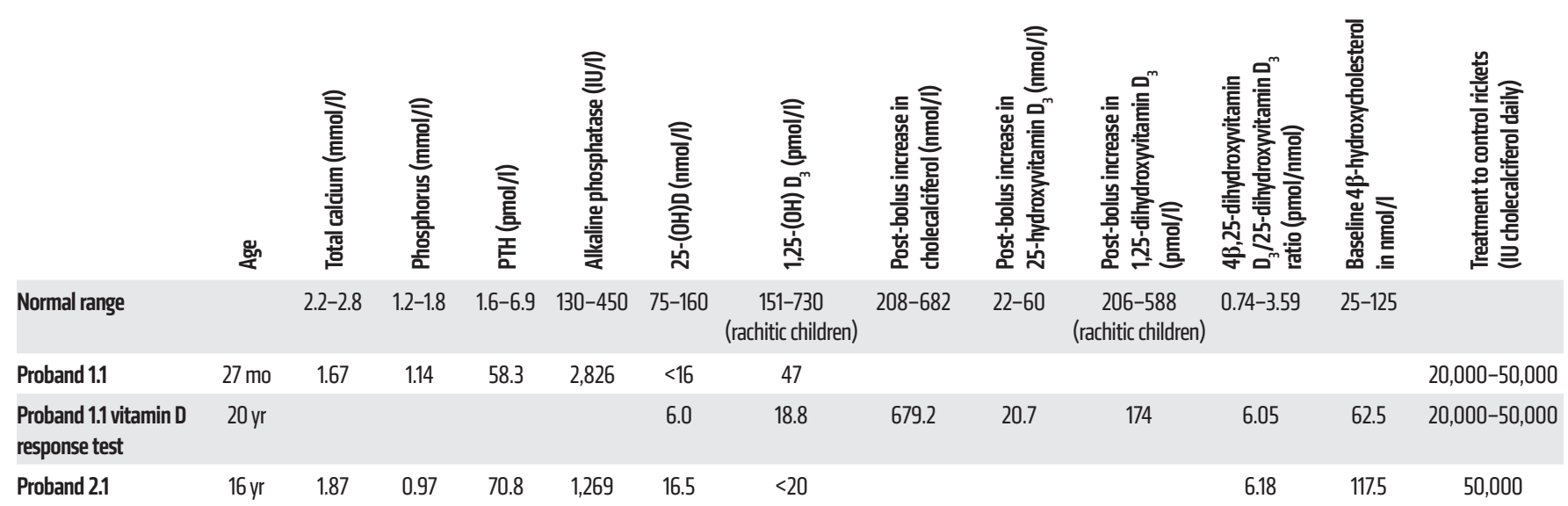

min $\mathrm{D}_{3}$, but blunted increases in serum 25-hydroxyvitamin $\mathrm{D}$ and 1,25-dihydroxyvitamin D compared with control subjects with nutritional vitamin D deficiency rickets (Table 1 and ref. 9). Moreover, serum 24,25-dihydroxyvitamin D was low (data not shown), suggesting that increased vitamin $\mathrm{D}$ metabolite inactivation by CYP24A1 did not account for apparent vitamin D deficiency. Both subjects required high-dose calcitriol or vitamin $\mathrm{D}_{3}(50,000 \mathrm{IU}$ daily) to maintain normal serum vitamin $\mathrm{D}$ metabolites, parathyroid hormone, calcium, and phosphorus. Neither child had a family history of rickets, and all available first-degree relatives had normal circulating vitamin D, metabolites, parathyroid hormone, calcium, and phosphorus (data not shown).

Targeted sequencing identified no mutations in known VDDR1 genes; however, whole exome sequencing revealed a recurrent mutation in CYP3A4. Nucleotide sequences including $5 \mathrm{~kb}$ upstream of the transcription start site, as well as exons and exon-intron boundaries for the CYP2R1, CYP27B1, VDR (proband 1.1), and CYP24A1 genes were normal. Whole exome sequencing was performed in probands and parents, and the data were analyzed using models for X-linked, autosomal recessive, and dominant modes of inheritance. No potential candidate gene with 2 mutations was identified; however, each subject had several heterozygous variants potentially causing protein-function alterations. To identify de novo dominant variants, we assumed that disease-causing variants would be rare. Supplemental Table 1 (supplemental material available online with this article; https://doi.org/10.1172/ JCI98680DS1) shows surviving candidate variants in the 2 subjects that were absent from their parents. Of these candidates, only CYP3A4 (GenBank NM_017460.5) showed a variant in both subjects, and remarkably, the subjects carried an identical heterozygous single nucleotide change $(\mathrm{c} .902 \mathrm{~T}>\mathrm{C})$ that results in replacement of isoleucine by threonine at codon 301 (p.I301T). Sanger sequencing confirmed that both subjects but no available relatives, who were unaffected, carried the missense mutation, indicating the mutation was both recurrent and de novo (Figure 1B). This mutation was not present in public databases or in data from more than 3,000 exomes analyzed at CHOP. Isoleucine 301 is highly conserved (Figure 1C) and forms a critical portion of substrate recognition site 4 (SRS-4), one of 6 SRSs determining CYP3A4 substrate selectivity and product profile (10). SIFT (http://sift.jcvi.org/) and MutPred (http://mutpred.mutdb.org/) predict this change would damage function, but MutPred additionally predicts a possible novel catalytic function. To examine the possibility of a common genetic origin of the mutation, we determined CYP3A4 gene haplotypes in both families. No common haplotype was shared by the 2 mutation carriers, excluding the possibility of a common founder.

Functional assessment of the CYP3A4 p.I301T mutant. Because previous work had shown that P.I301 is a determinant of activity for CYP3A4 SRS-4 substrates (11), we hypothesized that threonine replacement of isoleucine 301 might increase oxidation of the vitamin D metabolites 25-hydroxyvitamin D and 1,25-dihydroxyvitamin D $(12,13)$. We tested this hypothesis by analyzing serum 4,25-dihydroxyvitamin D, the principal product of CYP3A4 metabolism of 25-hydroxyvitamin D $(14,15)$. In both subjects, the ratio of $4 \beta, 25$-dihydroxyvitamin $\mathrm{D}$ to 25 -dihydroxyvitamin $\mathrm{D}$ was markedly elevated to the range of values exhibited by patients taking rifampin (16), an inducer of CYP3A4 (Table 1). We next used a mammalian cell 2-hybrid expression system in which intracellular 1,25-dihydroxyvitamin stimulates transcription of a luciferase reporter gene (4) to assess the ability of the mutant recombinant CYP3A4 protein to inactivate 1,25 -dihydroxyvitamin $\mathrm{D}_{3}$. Cells expressing mutant compared with WT recombinant CYP3A4 protein had decreased luciferase activity, indicating that p.1301T possessed greater ability to inactivate 1,25 -dihydroxyvitamin $D_{3}$ (Figure 2A) than WT $(P<0.01$ for overall curves; post hoc multiple comparison-adjusted analyses confirmed significance at $0.03 \mathrm{ng} / \mathrm{ml}$ [mean difference: 0.13; 95\% CI, 0.08-0.22], $0.1 \mathrm{ng} /$ $\mathrm{ml}$ [mean difference: 0.72; 95\% CI, 0.25-1.20], $0.3 \mathrm{ng} / \mathrm{ml}$ [mean difference: 2.03 ; 95\% CI, 1.55-2.51], and $1 \mathrm{ng} / \mathrm{ml}$ [mean difference: 3.6 ; 95\% CI, 3.1-4.1]; $P<0.01$ for each). Moreover, p.I301T CYP3A4 was more active than CYP24A1, the principal inactivator of 1,25-dihydroxyvitamin $\mathrm{D}_{3}$, at $0.3 \mathrm{ng} / \mathrm{ml}$ (mean difference: 0.42 ; 95\% CI, 0.06-0.77; $P<0.05$ ). We also compared apparent kinetics data between enzymes, as measurements were performed using reporter activity in whole cells. The apparent catalytic efficiency 
A

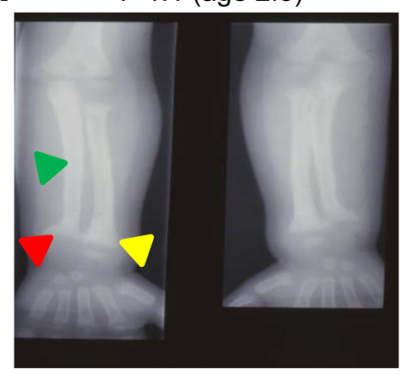

P 2.1 (age 16)

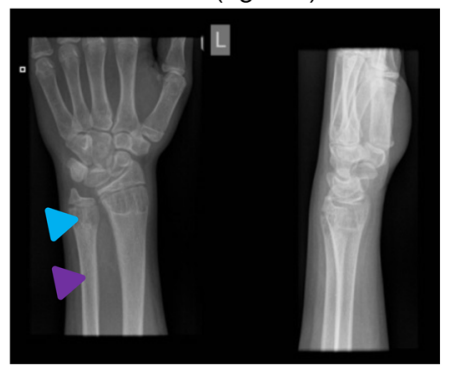

C

$\begin{array}{llll}\text { Human 3A4 } & \text { ALSDLELVAQSI } & \text { I } & \text { FIFAGYETTS } \\ \text { Rhesus } & \text { ALSDLELVAQSI } & \text { I } & \text { FIFAGHETTS } \\ \text { Mouse } & \text { ALSDVEIVAQSV } & \text { I FIFAGYETTS } \\ \text { Dog } & \text { ALSDLELVAQS } & \text { I FIFAGYTTS } \\ \text { Elephant } & \text { ALTDTEPVAQSF } & \text { I FILAGYETTS } \\ \text { Chicken } & \text { ALSDIEVLSQAF } & \text { I FIFAGYETS } \\ \text { X_tropic } & \text { KLSDTEILAQSI } & \text { I FIMAGYTTS } \\ \text { Zebrafish } & \text { RLTDHEILSQSF } & \text { I FILGGYTTS } \\ \text { Human 3A5 } & \text { ALSDLELAASI } & \text { I FIFAGYTTS } \\ \text { Human 3A7 } & \text { ALSDLELMAQSI } & \text { I FIFAGYETTS } \\ \text { Human 3A43 } & \text { ALSDLELVAQSI } & \text { I } & \text { IIFAAYTTS }\end{array}$

B Family 1
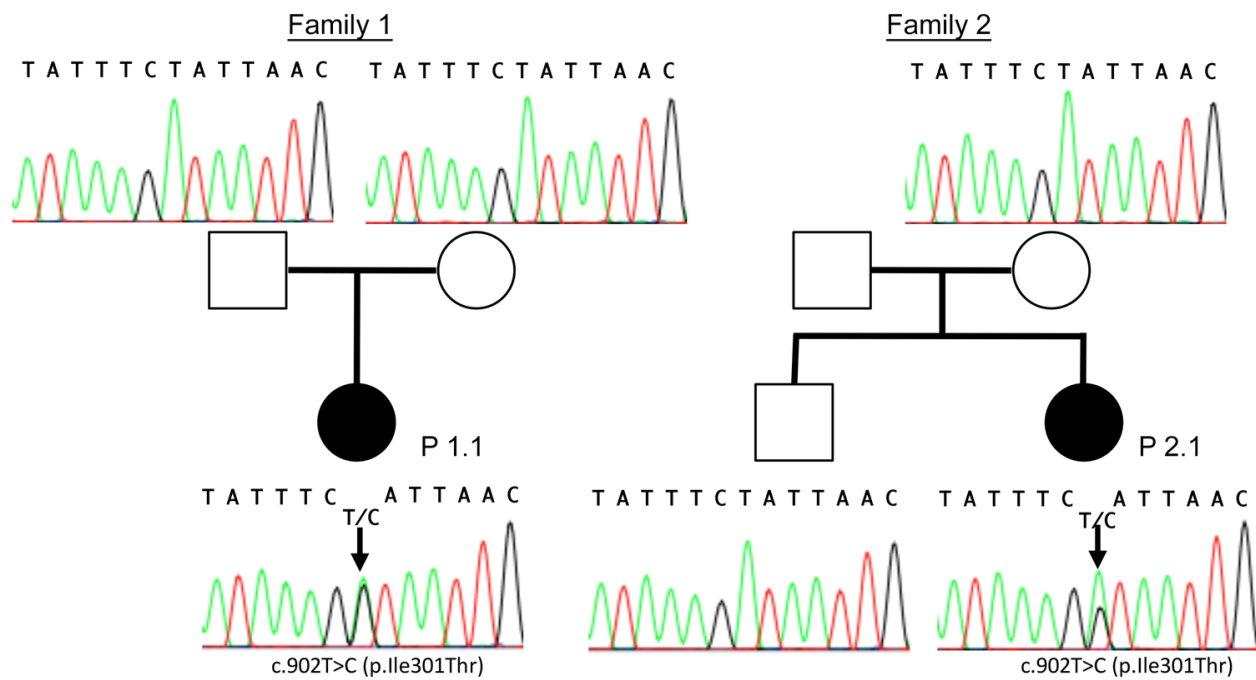

Figure 1. CYP3A4 mutation causes VDDR. (A) Wrist radiographs of the 2 probands are consistent with those of untreated rickets despite highdose supplementation. Wrist radiographs from these patients show the typical features of rickets: younger growing patients have bowing (green triangle), metaphyseal splaying (yellow triangle), and cupping (red triangle), as in proband 1.1 (P 1.1); older patients have metaphyseal lucency (blue triangle) and osteopenia (purple triangle), as in proband 2.1; features change as the role of calcification changes with age (supporting growth vs. maintaining bone integrity). (B) Family pedigrees are consistent with p.I301T mutations arising de novo. Family pedigrees of the 2 probands with Sanger sequencing results of the mutation region in CYP3A4 from all available family members. (C) Alignment of CYP3A4 protein sequence surrounding p.I301T mutation reveals high conservation of this residue across species. Isoleucine 301 is highly conserved in CYP3A4 across evolution and within the human $3 \mathrm{~A}$ protein family.

$\left[V_{\max }(\mathrm{app}) / K_{m}(\mathrm{app})\right]$ of the WT CYP3A4 was 0.014, while that of the p.I301T CYP3A4 mutant was 0.14 and that of CYP24A1 was 0.08. Thus, p.I301T was nearly 10 -fold more active than the WT and nearly twice as active as CYP24A1. Because both the WT and mutant CYP3A4 showed increased activity when cotransfected with a cDNA encoding $\mathrm{P} 450$ oxoreductase, which localizes to the ER, we believe that the c.I301T substitution does not prevent proper ER localization of CYP3A4.

Increased activity of CYP3A 4 p.I301T in transfected cells is not due to increased recombinant protein abundance. To determine whether the increased in vitro activity of p.I301T mutant CYP3A4 might be due to increased protein abundance, we measured recombinant CYP3A4 proteins levels by semiquantitative immunoblotting. The size and abundance of the p.I301T mutant protein were similar to those of the WT (Figure 2, B and C).

CYP3A4 p.I301T does not have increased activity for non-vitamin D substrates. CYP3A4 has 6 substrate domains (10), and the replacement of isoleucine 301 by threonine is predicted to affect only SRS4 substrates. To assay the effect of p.I301T on non-vitamin D substrates, we first examined circulating $4 \beta$-hydroxycholes- terol, as this endogenous oxysterol is formed from cholesterol by CYP3A4 and is commonly used as a marker for CYP3A4 activity (17). Both subjects had normal circulating $4 \beta$-hydroxycholesterol (Table 1 and ref. 18). We next used a cell-based assay to assess p.I301T CYP3A4 activity for the alternative substrate luciferin IPA. Because the kidney cell line (HEK293T) does not express CYP3A4, we used it to create a cell-based assay. This assay directly measures the conversion of luciferin IPA to luciferin by CYP3A4 and is used to examine CYP3A4 induction (19). Enzymatic activity of both the WT and p.I301T mutant were inhibited by ketoconazole to levels indistinguishable from empty vector (Figure $2 \mathrm{D}: 1.1 \pm 0.1$ vs. $1.0 \pm 0.1, n=4$ for each, $P=0.39$ ). In contrast to the results for 1,25 -dihydroxyvitamin $\mathrm{D}_{3}$ as a substrate, the p.I301T mutant had significantly decreased activity relative to the WT (Figure 2D: $30.0 \pm 1.6$ vs. $50.1 \pm 1.6, n=4$ for each, $P<0.001$ ) for luciferin IPA (19).

We identified a gain-of-function mutation in CYP3A4 in 2 unrelated children with severe rickets due to accelerated vitamin D metabolite inactivation. The observation of identical mutations in the patients, who do not share CYP3A4 haplotypes, makes a founder 
A

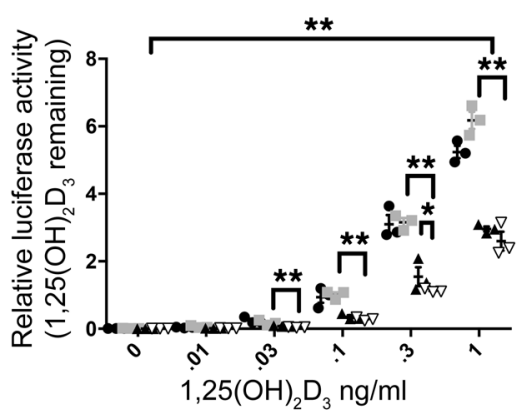

C

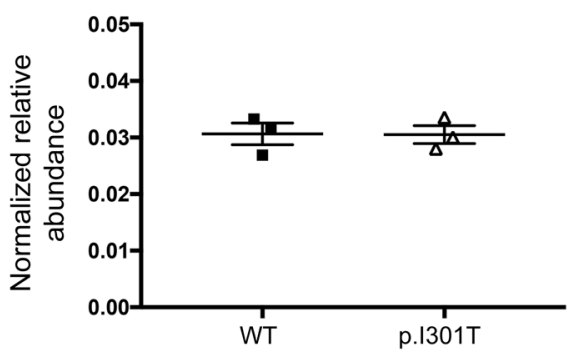

- Empty vector - 1,25(OH)D serial dilution

- CYP3A4 WT - 1,25(OH)D serial dilution

- CYP24A1 - 1,25(OH)D serial dilution

$\nabla$ CYP3A4 mutant - 1,25(OH)D serial dilution
B

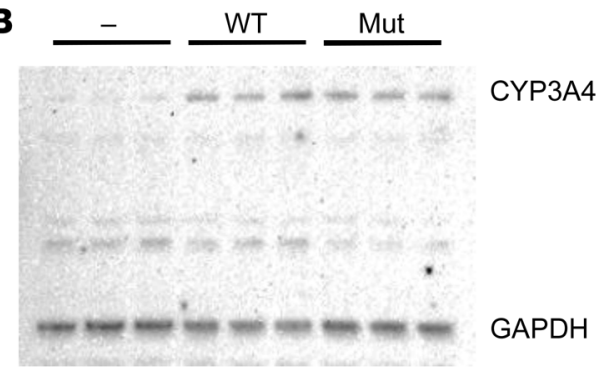

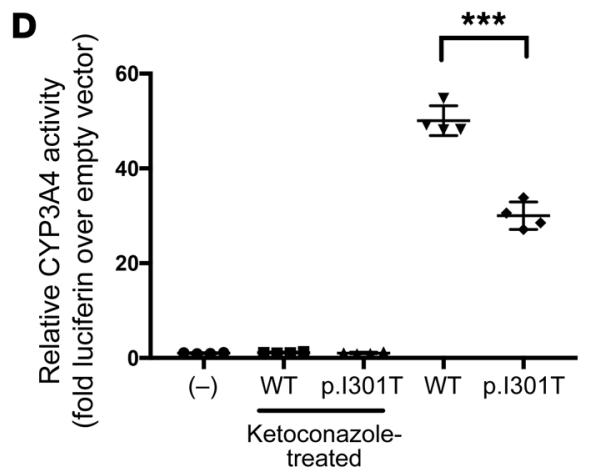

Figure 2. CYP3A4 (p.I301T) mutant has increased vitamin D degradative activity but decreased activity for other substrates. (A) CYP3A4 (p.I301T) has increased vitamin D degradative activity. CYP3A4 $p$. I301T had increased inactivation of calcitriol relative to WT ( ${ }^{* *} P<0.01$ by 2 -way ANOVA for curve), and post hoc multiple comparison-adjusted analyses confirmed significant differences at $0.03,0.1$, and $0.3 \mathrm{ng} / \mathrm{ml}\left({ }^{* *} P<0.01\right)$, and relative to $C Y P 24 \mathrm{~A} 1$ at $0.3 \mathrm{ng} / \mathrm{ml}\left({ }^{*} P<0.05\right)(n=3$ for each). (B and C) There is no difference in the relative abundance of the WT and p.I301T CYP3A4 ( $n=3$ for each treatment). (B) Immunoblot of cell lysates for CYP3A4 and GAPDH. (C) Quantification of the relative abundance of CYP3A4 and GAPDH. No significant differences were observed between the abundance of the WT and the p.I301T mutant. (D) CYP3A4 (p.I301T) does not have increased catalytic activity for non-vitamin D substrates. The p.I301T mutant had significantly decreased activity for luciferin IPA relative to the WT enzyme (30.0 \pm 1.6 vs. $50.1 \pm 1.6, n=4$ for each, $\left.{ }^{* * *} P<0.001\right)$. ${ }^{* *} P<0.05$. Data are presented as mean \pm SEM.

mutation unlikely and is most consistent with recurrent independent mutation events. CYP3A4 (Enzyme Commission [EC] 1.14.13.97) is the most abundant P450 in the liver and is also highly expressed in the intestine. CYP3A4 serves an important metabolic role in biotransforming a wide variety of compounds, including many drugs, steroids, xenobiotics, and carcinogens. Most relevant to this work, CYP3A4 can oxidize and inactivate the vitamin D metabolites 25-hydroxvitamin D and1,25-dihydroxyvitamin D (15). Although the impact of CYP3A4 on vitamin D homeostasis is limited under physiological conditions, under special circumstances CYP3A4 can have significant effects on vitamin D metabolism. For example, induction of CYP3A4 by anticonvulsants is associated with vitamin D deficiency (15). In addition, targeted induction of CYP3A4 by rifampin provides an alternative clearance mechanism

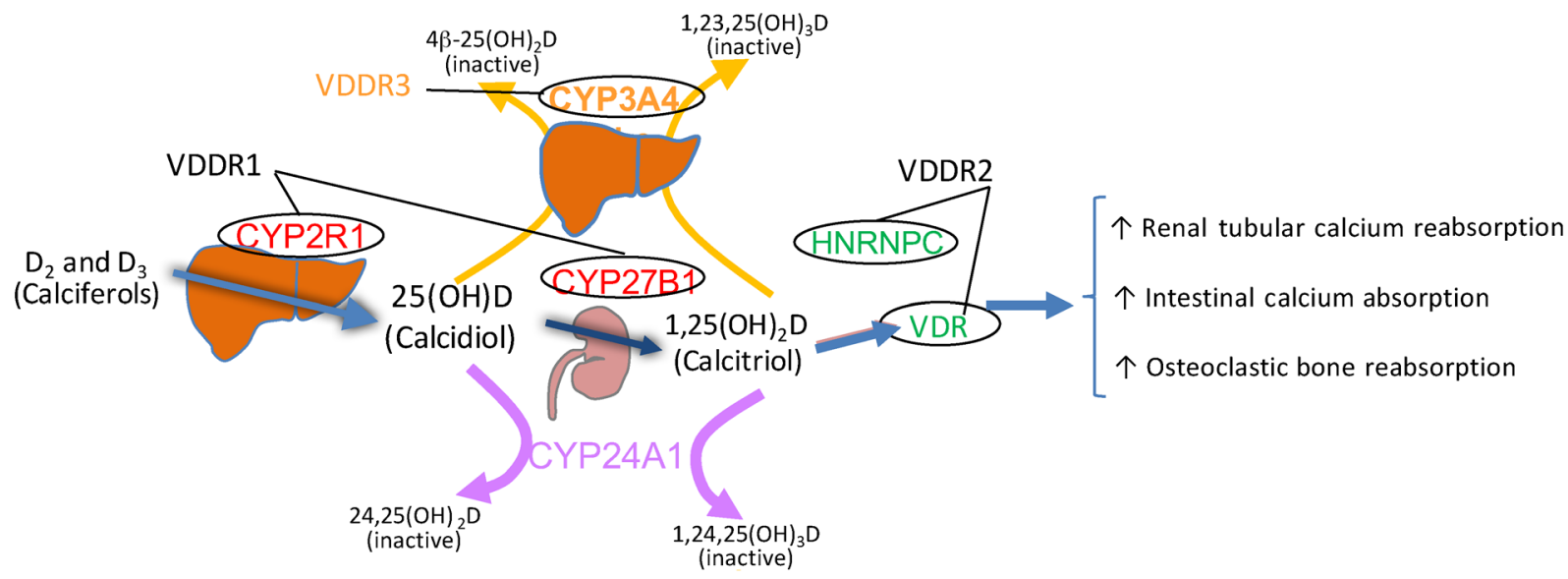

Figure 3. Pathogenesis of VDDR. VDDR-1s are caused by mutations in genes encoding proteins that activate vitamin D: CYP2R1 and CYP27B1. VDDR-2s are caused by mutations in genes encoding signal transducing proteins: VDR and HNRNPC. Type 3 is due to gain-of-function mutations in a gene encoding a vitamin D-degrading enzyme: CYP3A4. 
that can normalize elevated vitamin D metabolites in patients who lack the native pathway for vitamin D oxidation due to loss-of-function mutations in CYP24A1 (16). Finally, common polymorphisms in CYP3A4 are associated with decreased bone density (20).

Several lines of evidence support our hypothesis that the p.I301T CYP3A4 mutation is the basis for a selective defect in vitamin D metabolism. First, the 2 affected subjects had elevated $4 \beta, 25$-dihydroxyvitamin $\mathrm{D} / 25$-hydroxyvitamin $\mathrm{D}$ ratios but normal 4 $\beta$-hydroxycholesterol. Second, molecular modeling and in vitro studies previously identified isoleucine 301 as an important determinant of substrate binding; and that replacing isoleucine 301 with another nonconservative residue, phenylalanine, results in 4-fold increases in CYP3A4 activity for some substrates $(10,14)$. Third, our in vitro studies showed that p.I301T CYP3A4 significantly increased inactivation of 1,25-dihydroxyvitamin $\mathrm{D}_{3}$ but had no effect on clearance of substrate luciferin IPA. Although we did not measure other steroid hormones in the 2 patients, there was no clinical evidence of additional endocrine defects. Therefore, the genetic gain of function in CYP3A4 recapitulated the selective inactivation of vitamin D metabolites well described in patients taking drugs that induce CYP3A4 (21).

These observations lead to us to propose that a gain-of-function mutation of CYP3A4 causes a distinct form of VDDR. In contrast to the previously described autosomal recessive forms of VDDR that result from defects in either synthesis of vitamin D metabolites or responsiveness to 1,25 -dihydroxyvitamin $\mathrm{D}$, this dominant form of VDDR is due to accelerated inactivation of vitamin D metabolites (schematic of VDDRs in Figure 3). Although nutritional vitamin $\mathrm{D}$ deficiency is the most common cause of childhood rickets, these 2 cases, representing the identification of a previously undescribed VDDR, emphasize the importance of considering accelerated vitamin $\mathrm{D}$ inactivation as a risk factor for vitamin D deficiency. There is renewed awareness of the importance of daily vitamin D prophylaxis for the prevention of vitamin D deficiency and rickets in infants and children, and our findings highlight the importance of genetic and induced variation in CYP3A4 activity as a modifier of the amount of vitamin D necessary to maintain vitamin $\mathrm{D}$ homeostasis.

\section{Methods}

For further information, see Supplemental Methods.

Biochemical and molecular analyses. We measured serum and urine electrolytes, serum creatinine, and parathyroid hormone using routine methods. Measurements of vitamin $\mathrm{D}_{3}$, vitamin $\mathrm{D}_{2}, 25$-hydroxyvitamin $\mathrm{D}_{3}$, 25-hydroxyvitamin $\mathrm{D}_{2}, 24,25$-dihydroxyvitamin $\mathrm{D}$, and $4 \beta$-hydroxycholesterol were performed by isotope dilution liquid chromatography-tandem mass spectrometry. We measured serum 1,25-dihydroxyvitamin $\mathrm{D}_{3}$ and 4 $\beta, 25$-dihydroxyvitamin $\mathrm{D}$ using an ultrahigh resolution chromatographic separation procedure (15) that enables their complete separation.
We performed whole-exome sequencing of DNA from proband 1.1, proband 2.1, and their available relatives (Figure 1), as described previously (22). Analysis of candidate genes and CYP3A4 confirmatory genotyping were performed by Sanger sequencing. Analysis of 1,25-dihydroxyvitamin D degradative activity of WT and mutant CYP3A4 recombinant proteins was performed using a mammalian 2-hybrid expression system in which activity of firefly luciferase is proportional to the concentration of intracellular 1,25-dihydroxyvitamin D (see Supplemental Methods and ref. 4). Activity against other substrates was assayed by measuring CYP3A4 conversion of luciferin IPA to the luminescent luciferin.

Cell lines. HEK293T cells were obtained from ATCC (catalog CRL-3216).

In vitro assessment of expression and activity of CYP3A4. Mutations were introduced by PCR site-directed mutagenesis into a full-length human CYP3A4 cDNA (provided by P.F. Hollenberg, University of Michigan; ref. 23).

Statistics. All assays were performed in biological quadruplicate. All statistical analyses were performed using GraphPad Prism 7. Sample means were compared using a $t$ test or ANOVA with Tukey's 2 -tailed post hoc test as appropriate, with a $P$ value of 0.05 defined as statistically significant. All data are presented as mean \pm SEM.

Study approval. All studies were approved by the institutional review board of the Children's Hospital of Philadelphia. Patients or parents provided written informed consent/assent prior to inclusion in the study.

\section{Author contributions}

JDR prepared the manuscript and contributed to the study design and data analyses. DL and $\mathrm{HH}$ performed and interpreted the whole exome sequence analyses. LO performed assays and collected data. MKJ, NJS, PRE, HHN, CPR, KET, and TDT contributed to study design, collection of clinical and biochemical data, and characterization of the subjects' phenotypes. MAL contributed to overall study design, data analysis, interpretation, and preparation of the manuscript. All authors reviewed and approved the final manuscript.

\section{Acknowledgments}

We thank Brian Phillips for help examining vitamin D metabolites and Harsh Kanwar for assistance in performing assays. This study was supported in part by grants from the NIH (R01 DK079970 and R01 DK112955 to MAL; K08 HD087964 to JDR), the Children's Hospital of Philadelphia Research Institute (to MAL), and the Institutional Development Fund to the Center for Applied Genomics from the Children's Hospital of Philadelphia (to HH). MKJ was supported by the National Institute of Health Research (NIHR) Oxford Biomedical Research Centre (BRC).

Address correspondence to: Michael A. Levine, Children's Hospital of Philadelphia, 3401 Civic Center Blvd., Philadelphia, Pennsylvania 19103, USA. Phone: 215.590.3907; Email: levinem@chop.edu.

\footnotetext{
1. Terushkin V, Bender A, Psaty EL, Engelsen O, Wang SQ, Halpern AC. Estimated equivalency of vitamin $\mathrm{D}$ production from natural sun exposure versus oral vitamin D supplementation across seasons at two US latitudes. J Am Acad Dermatol. 2010;62(6):929.e1-929.e9.
}

2. Kitanaka S, et al. Inactivating mutations in the 25-hydroxyvitamin D3 1alpha-hydroxylase gene in patients with pseudovitamin D-deficiency rickets. N Engl J Med.1998;338(10):653-661.

3. Cheng JB, Levine MA, Bell NH, Mangelsdorf DJ, Russell DW. Genetic evidence that the human CYP2R1 enzyme is a key vitamin D 25-hydroxylase. Proc Natl Acad Sci U S A 2004;101(20):7711-7715.

4. Thacher TD, Fischer PR, Singh RJ, Roizen J, Levine MA. CYP2R1 mutations impair generation of 25-hydroxyvitamin D and cause an atyp- 
ical form of vitamin D deficiency. J Clin Endocrinol Metab. 2015;100(7):E1005-E1013.

5. Chen H, Hewison M, Hu B, Adams JS. Heterogeneous nuclear ribonucleoprotein (hnRNP) binding to hormone response elements: a cause of vitamin D resistance. Proc Natl Acad Sci U S A. 2003;100(10):6109-6114.

6. Pike JW, Dokoh S, Haussler MR, Liberman UA, Marx SJ, Eil C. Vitamin D3-resistant fibroblasts have immunoassayable 1,25-dihydroxyvitamin D3 receptors. Science. 1984;224(4651):879-881.

7. Makin G, Lohnes D, Byford V, Ray R, Jones G. Target cell metabolism of 1,25-dihydroxyvitamin D3 to calcitroic acid. Evidence for a pathway in kidney and bone involving 24-oxidation. Biochem J. 1989;262(1):173-180.

8. Sakaki T, et al. Dual metabolic pathway of 25-hydroxyvitamin D3 catalyzed by human CYP24. Eur J Biochem. 2000;267(20):6158-6165.

9. Thacher TD, Fischer PR, Obadofin MO, Levine MA, Singh RJ, Pettifor JM. Comparison of metabolism of vitamins D2 and D3 in children with nutritional rickets. JBone Miner Res. 2010;25(9):1988-1995.

10. Szklarz GD, Halpert JR. Molecular modeling of cytochrome P450 3A4. JComput Aided Mol Des. 1997;11(3):265-272.

11. Khan KK, Halpert JR. Structure-function analysis of human cytochrome P450 3A4 using 7-alkoxy- coumarins as active-site probes. Arch Biochem Biophys. 2000;373(2):335-345.

12. Wang Z, et al. An inducible cytochrome P450 3A4-dependent vitamin D catabolic pathway. Mol Pharmacol. 2012;81(4):498-509.

13. Zheng XE, et al. Human PXR-mediated induction of intestinal CYP3A4 attenuates 1 $\alpha, 25$-dihydroxyvitamin $\mathrm{D}$ (3) function in human colon adenocarcinoma LS180 cells. Biochem Pharmacol. 2012;84(3):391-401.

14. Gupta RP, He YA, Patrick KS, Halpert JR, Bell NH. CYP3A4 is a vitamin D-24- and 25-hydroxylase: analysis of structure function by site-directed mutagenesis. JClin Endocrinol Metab. 2005;90(2):1210-1219.

15. Wang Z, et al. Simultaneous measurement of plasma vitamin $\mathrm{D}(3)$ metabolites, including $4 \beta, 25$-dihydroxyvitamin $\mathrm{D}(3)$, using liquid chromatography-tandem mass spectrometry. Anal Biochem. 2011;418(1):126-133.

16. Hawkes CP, Li D, Hakonarson H, Meyers KE, Thummel KE, Levine MA. CYP3A4 induction by Rifampin: an alternative pathway for vitamin $D$ inactivation in patients with CYP24A1 mutations. JClin Endocrinol Metab. 2017;102(5):1440-1446.

17. Dutreix C, Lorenzo S, Wang Y. Comparison of two endogenous biomarkers of CYP3A4 activity in a drug-drug interaction study between midostaurin and rifampicin. Eur JClin Pharmacol.
2014;70(8):915-920.

18. Diczfalusy U, et al. $4 \beta$-hydroxycholesterol is a new endogenous CYP3A marker: relationship to CYP3A5 genotype, quinine 3-hydroxylation and sex in Koreans, Swedes and Tanzanians. Pharmacogenet Genomics. 2008;18(3):201-208.

19. Kuzbari O, et al. Comparative analysis of human CYP3A4 and rat CYP3A1 induction and relevant gene expression by bisphenol A and diethylstilbestrol: implications for toxicity testing paradigms. Reprod Toxicol. 2013;37:24-30.

20. Kang YS, et al. The CYP3A $4 * 18$ genotype in the cytochrome $\mathrm{P} 4503 \mathrm{~A} 4$ gene, a rapid metabolizer of sex steroids, is associated with low bone mineral density. Clin Pharmacol Ther. 2009;85(3):312-318.

21. Wang Z, et al. Enhancement of hepatic 4-hydroxylation of 25-hydroxyvitamin D3 through CYP3A4 induction in vitro and in vivo: implications for drug-induced osteomalacia. J Bone Miner Res. 2013;28(5):1101-1116.

22. Li D, et al. Autosomal dominant hypoparathyroidism caused by germline mutation in GNA11: phenotypic and molecular characterization. J Clin Endocrinol Metab. 2014;99(9):E1774-E1783.

23. Pratt-Hyatt M, Zhang H, Snider NT, Hollenberg PF. Effects of a commonly occurring genetic polymorphism of human CYP3A4 (I118V) on the metabolism of anandamide. Drug Metab Dispos. 2010;38(11):2075-2082. 